\title{
Incidence of febrile seizures and associated factors in children in Soweto, South Africa
}

\author{
N D Tebeila, ${ }^{1,2}$ MSc (Epi); Z Dangor, ${ }^{1,3} \mathrm{PhD}$; S A Madhi, ${ }^{1,4} \mathrm{PhD}$; C Cutland, ${ }^{1,4,5} \mathrm{PhD}$; M J Groome, ${ }^{1,4} \mathrm{PhD}$ \\ ${ }^{1}$ South African Medical Research Council Vaccines and Infectious Diseases Analytics Research Unit, Faculty of Health Sciences, University of the \\ Witwatersrand, Johannesburg, South Africa \\ ${ }^{2}$ Division of Epidemiology and Biostatistics, School of Public Health, Faculty of Health Sciences, University of the Witwatersrand, Johannesburg, \\ South Africa \\ ${ }^{3}$ Department of Paediatrics and Child Health, Faculty of Health Sciences, University of the Witwatersrand, Johannesburg, South Africa \\ ${ }^{4}$ Department of Science and Technology/National Research Foundation: Vaccine Preventable Diseases, Faculty of Health Sciences, University of \\ the Witwatersrand, Johannesburg, South Africa \\ ${ }^{5}$ African Leadership in Vaccinology Expertise (ALIVE), School of Pathology, Faculty of Health Sciences, University of the Witwatersrand, \\ Johannesburg, South Africa
}

Corresponding author: N D Tebeila (naumetebeila@gmail.com/tebeiln@rmpru.co.za)

\begin{abstract}
Background. Febrile seizures (FSs) are a common cause of paediatric emergencies, but there is limited research on the aetiology and epidemiology of FSs, especially in Africa.

Objectives. To determine the incidence of FS hospitalisations in children aged 6 - 59 months in Soweto, South Africa, and factors associated with FS hospitalisations.

Method. In a secondary data analysis using a cohort of children enrolled in a 9-valent pneumococcal conjugate vaccine efficacy trial conducted in Soweto during 1998 - 2005, the incidence of FS hospitalisation was calculated and stratified by age group. Regression analysis was used to investigate factors associated with FS at the time of hospitalisation. Influenza A, influenza B, respiratory syncytial virus (RSV), adenovirus and parainfluenza were investigated for among those with respiratory symptoms using immunofluorescent assays.

Results. FSs accounted for $780(11.0 \%)$ of 7126 hospitalisations during the study period. The overall incidence of FSs was 4.4 (95\% confidence interval (CI) 4.10 - 4.97) per 1000 person-years, with the highest incidence in children aged 12 - 23 months (7.25; $95 \%$ CI 6.44 8.14). Among hospitalised children, FS hospitalisation was associated with HIV-negative status (odds ratio (OR) 6.25; $95 \%$ CI 4.34 - 8.99 ), body temperature $\geq 39^{\circ} \mathrm{C}$ (OR 2.03; 95\% CI 1.56 - 2.64) and concurrent diagnosis of acute otitis media (OR 2.16; 95\% CI 1.74 - 2.67). Influenza A was identified in 44/515 FS hospitalisations (8.5\%) compared with 123/3 794 non-FS hospitalisations (3.2\%) (OR 2.22; 95\% CI 1.56 - 3.16). In contrast, RSV detection was less commonly identified in children with FSs $(21 ; 4.1 \%)$ than without (419; $11.0 \%)($ OR 0.36 ; 95\% CI $0.24-0.54)$.

Conclusions. FSs contributed significantly to the burden of paediatric hospitalisations in Soweto, and were strongly associated with influenza A virus infection.
\end{abstract}

S Afr Med J 2021;111(8):796-802. https://doi.org/10.7196/SAMJ.2021.v111i8.15431

Febrile seizures (FSs) account for $48-62 \%$ of seizure types in paediatric emergency departments, ${ }^{[1-3]}$ making them the most frequent type of acute seizures affecting children aged $<5$ years. ${ }^{[4]}$ FSs are defined by the American Academy of Paediatrics (AAP) as a seizure occurring in febrile children aged 6 - 60 months, without intracranial infections, metabolic disturbances or a history of afebrile seizures. ${ }^{[5]}$ The aetiology of FSs is not well understood; however, a genetic predisposition may play a role. ${ }^{[6,7]}$ Most FSs are provoked by febrile illnesses associated with certain viral infections such as human herpesvirus-6 (HHV-6), rotavirus causing gastroenteritis, and influenza A respiratory infections. ${ }^{[8-10]}$

Although generally benign, FSs require urgent medical attention and significantly contribute to the burden of paediatric emergency visits at health facilities worldwide. Furthermore, FSs are a terrifying experience for parents and cause them significant anxiety. ${ }^{[1,12]}$ It is estimated that 2 - 5\% of children in Europe and North America will visit a paediatric emergency department for a FS before the age of 5 years; ${ }^{[13,14]}$ estimates in Asia are reportedly higher at $8-10 \% .{ }^{[15]}$ However, epidemiological studies describing FSs in Africa are limited, and the inclusion of acute afebrile or unprovoked childhood seizures as well as an inconsistent age definition often confound the estimates. ${ }^{[16-19]}$

\section{Objectives}

To determine the incidence of FS hospitalisations in children 6 - 59 months of age in Soweto, South Africa (SA), as well as to investigate factors associated with FS hospitalisations.

\section{Methods}

\section{Study design and population}

This study was a secondary analysis of data derived from a cohort of 39836 children enrolled into a phase III trial evaluating the efficacy of a 9-valent pneumococcal conjugate vaccine (PCV-9) conducted in Soweto, as described. ${ }^{[20]}$ Healthy children were enrolled at 6 weeks of age, from 2 March 1998 until 30 October 2000. Children were excluded if they had neurological disorders or a history of seizures, or if they were likely to move out of Soweto during the study period. Follow-up for hospitalisation was conducted by means of 24-hour hospital-based surveillance by study staff in the Chris Hani Baragwanath Academic Hospital (CHBAH) paediatric wards until 
October 2005. CHBAH is a secondary-tertiary hospital that serves $\sim 90 \%$ of children from Soweto requiring hospitalisation. Children enrolled in the trial who were hospitalised during the study period were examined by study doctors who completed a case report form recording patient demographics, clinical signs, symptoms and outcome. Children suspected to have lower respiratory tract infections (LRTIs) had blood taken for culture, chest radiographs performed, collection of nasopharyngeal aspirates (NPAs) and were tested for HIV. Respiratory viruses were identified from NPAs using immunofluorescent assays detecting influenza A, influenza $\mathrm{B}$, respiratory syncytial virus (RSV), adenovirus and parainfluenza. HIV testing was done through enzyme-linked immunosorbent assays in children aged $\geq 18$ months and by polymerase chain reaction in children aged $<18$ months. Study doctors did not take part in the management and care of the children after diagnosis.

The standard care for children hospitalised for FSs at CHBAH included establishing the cause of the fever and excluding intracranial or biochemical causes, e.g. any signs of meningitis. Antiepileptic medication would have been given if the child was still convulsing on arrival at the hospital. A lumbar puncture would have been done on some children with FSs to exclude bacterial meningitis. Children would have been hospitalised at least overnight for observation. Investigations involving skull radiographs, brain computed tomography scans, magnetic resonance imaging and electroencephalograms were not routinely performed.

Ethical approval for this secondary analysis was obtained from the Human Research Ethics Committee (Medical) at the University of the Witwatersrand, Johannesburg (ref. no. M180148).

\section{Data analyses}

Hospitalisations for FSs were identified using the International Classification of Diseases and Related Health Problems, 9th revision (ICD-9), codes in use at the time of the primary study. Using the AAP guidelines, any hospitalisation occurring from ages 6 to 59 months with either a primary or secondary discharge diagnosis of an ICD-9 coding of 780.3 was included. Multiple hospitalisations for FSs for the same child were included as separate events, provided that the episode occurred 14 days after the previous episode. Age at hospitalisation was categorised into the following groups: $6-11,12-23,24-35$ and 36 - 59 months. Gestational age was categorised as $<37$ weeks (preterm) or $\geq 37$ weeks (term). The maximum body temperature was measured as an axillary temperature and categorised into $<39^{\circ} \mathrm{C}$ and $\geq 39^{\circ} \mathrm{C}$. Acute otitis media was recorded as a dichotomous variable. Children with unknown HIV status were assumed to be negative based on the assumption that if they were positive they would have presented with clinical signs and therefore been tested at some point during the 5 years of follow-up.

Incidence was calculated by person-time analysis and included the full cohort of children enrolled and randomised. The person time contributed by each child was calculated from the age of 6 months and censored on the 5th birthday or when the child died, whichever occurred first. Each child only contributed once to the total person time. The numerator was the total number of FS hospitalisations that occurred during the time at risk, and the denominator was the total person time contributed by enrolled children. Rates were expressed per 1000 person-years with 95\% confidence intervals (CIs). Incidence rates were stratified by age group and treatment group (9-valent pneumococcal conjugate vaccine (PVC-9) and placebo; per protocol population).

A subset of the cohort, those who were hospitalised during the follow-up period, was used to investigate factors associated with FS at the point of hospitalisation using univariable and multivariable logistic regression analysis. Factors that were investigated included vaccination status (PVC-9 or placebo), sex, gestational age, maximum body temperature, presence of acute otitis media, HIV status and age at hospitalisation. Crude odds ratios (ORs) and adjusted ORs were determined, and $p$-values $\leq 0.05$ were considered significant.

Not all hospitalised participants had NPAs done for viral identification, so the association between these pathogens and FS hospitalisation was investigated separately from the main model through univariable logistic regression models. The number of influenza A hospitalisations throughout the study period was plotted together with the number of FS hospitalisations to investigate for temporal association between the two. All regression models were fitted using generalised estimating equations (GEEs) ${ }^{[21]}$ to account for the correlation resulting from multiple hospitalisations in the same participant. Data were analysed using Stata 14.0 (StataCorp, USA).

\section{Results}

\section{Description of hospitalisations}

A total of 10149 hospitalisations occurred among 39830 children during the follow-up period, with 7126 hospitalisations in children aged 6 - 59 months (Fig. 1). Of the 7126 children hospitalised, $780(11.0 \%)$ had FSs, including $387 / 3525(11.0 \%)$ in the PCV-9 group and $393 / 3601(10.9 \%)$ in the placebo group. Seventy-nine FS episodes (10.1\%) occurred in the age group 6 - 11 months, 287 $(36.8 \%)$ in the age group $12-23$ months, $233(29.9 \%)$ in the age group 24 - 35 months and 181 (23.2\%) in the age group 36 - 59 months. Of the 780 children with FSs, 463 (59.4\%) had lumbar punctures done and none had bacterial meningitis. The median (interquartile range) age of children with FSs was 25.0 (17.2 - 34.8) months. Of the FS hospitalisations, 325 (41.7\%) were of females and 454 (58.3\%) of males $(n=779)$. The majority $(71.9 \% ; n=561)$ of children diagnosed with FS were hospitalised for a median of 1 day (range 0 - 13).

Of the children with FS hospitalisation, $14.3 \%(n=92 / 645)$ went on to experience two or more episodes. Three or more FS episodes during the follow-up period were only observed in children who had their first FS before 36 months of age (Table 1).

Table 1. Number of children hospitalised for FSs stratified by age at first FS hospitalisation among children 6 - 59 months of age in Soweto, South Africa

\begin{tabular}{lllllll}
\hline & \multicolumn{5}{c}{ Number of FSs, $\boldsymbol{n}$ (\%) } \\
\cline { 2 - 7 } Age at first FS (months) & 1st & 2nd & 3rd & 4th & 5th & Total \\
\hline $6-11$ & $54(74.0)$ & $9(12.3)$ & $7(9.6)$ & $2(2.7)$ & $1(1.4)$ & 73 \\
$12-23$ & $201(82.7)$ & $27(1.1)$ & $9(3.7)$ & $5(2.1)$ & $1(0.4)$ & 243 \\
$24-35$ & $167(87.9)$ & $19(10.0)$ & $2(1.1)$ & $1(0.5)$ & $1(0.5)$ & 190 \\
$36-59$ & $131(94.2)$ & $8(5.8)$ & 0 & 0 & 0 & 139 \\
Total & $553(85.7)$ & $63(9.8)$ & $18(2.8)$ & $8(1.2)$ & $3(0.5)$ & 645 \\
FS/s = febrile seizure/s. & & & & & &
\end{tabular}




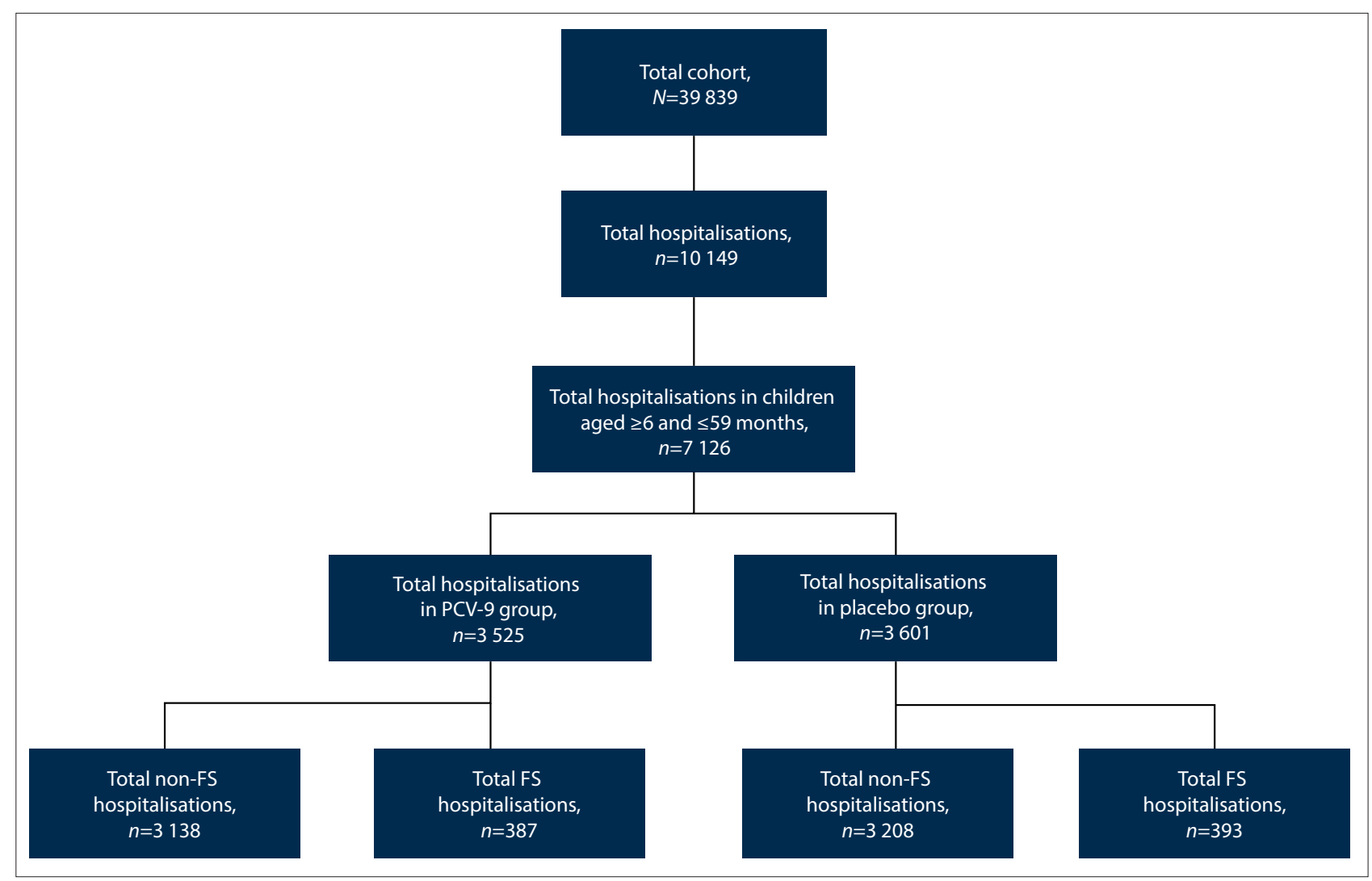

Fig. 1. Distribution of hospitalisation events among children enrolled in a randomised controlled trial in Soweto, South Africa. Only randomised children were included. (PCV-9 = 9-valent pneumococcal conjugate vaccine; $F S=$ febrile seizure. $)$

\section{Incidence of FS hospitalisations}

The overall incidence of FS hospitalisations occurring in children aged 6 - 59 months was 4.40 (95\% CI 4.10 - 4.97) per 1000 person-years of follow-up. Incidence rates were highest in the age groups 12 - 23 months and $24-35$ months, with rates of $7.25(95 \%$ CI 6.44 - 8.14) and 5.93 (95\% CI 5.20 - 6.72) per 1000 person-years, respectively (Fig. 2). The lowest incidence was observed in the age group 36 - 59 months $(2.29 ; 95 \%$ CI 1.96 - 2.64). The incidence of FS hospitalisations in the PCV-9 group (4.34; 95\% CI 3.92 - 4.78) did not differ in comparison with placebo recipients (4.48; 95\% CI 4.05 - 4.93).

\section{Factors associated with FS hospitalisation}

Among the hospitalised children, FSs were five times more common in the $24-35$ months age group than in those $6-11$ months old (OR 5.08; 95\% CI 3.87 - 6.67 (Table 2). Testing HIV-negative was also positively associated with FS hospitalisation (OR 6.25; 95\% CI 4.34 - 8.99), as was a temperature $\geq 39^{\circ} \mathrm{C}$ (OR 2.03 ; 95\% CI 1.56 2.64) and a diagnosis of acute otitis media (OR 2.16; 95\% CI 1.74 2.67). No significant associations were observed between sex, PCV-9 vaccination or gestational age and FS hospitalisation.

\section{Respiratory viruses and FS hospitalisations}

NPAs were performed in 4309 of the 7126 hospitalisations to investigate for respiratory virus infections. Viruses were identified from $18.7 \%$ ( $n=804 / 4309)$ children, of which $54.7 \%(n=440 / 804)$ were RSV, 20.8\% ( $n=167 / 804)$ influenza A, 1.0\% ( $n=3 / 804)$ influenza B, $13.9 \%(n=112 / 804)$ parainfluenza and $10.2 \%(n=82 / 804)$ adenovirus. Among children with FS, influenza A accounted for 48.4\% ( $n=44 / 91)$ of viruses identified, followed by RSV (23.1\%; $n=21 / 91)$, adenovirus $(18.7 \% ; n=17 / 91)$, parainfluenza $(8.8 \% ; n=8 / 91)$ and influenza B (1.1\%; $n=1 / 91)$ (Fig. 3A). Among non-FS hospitalisations, RSV

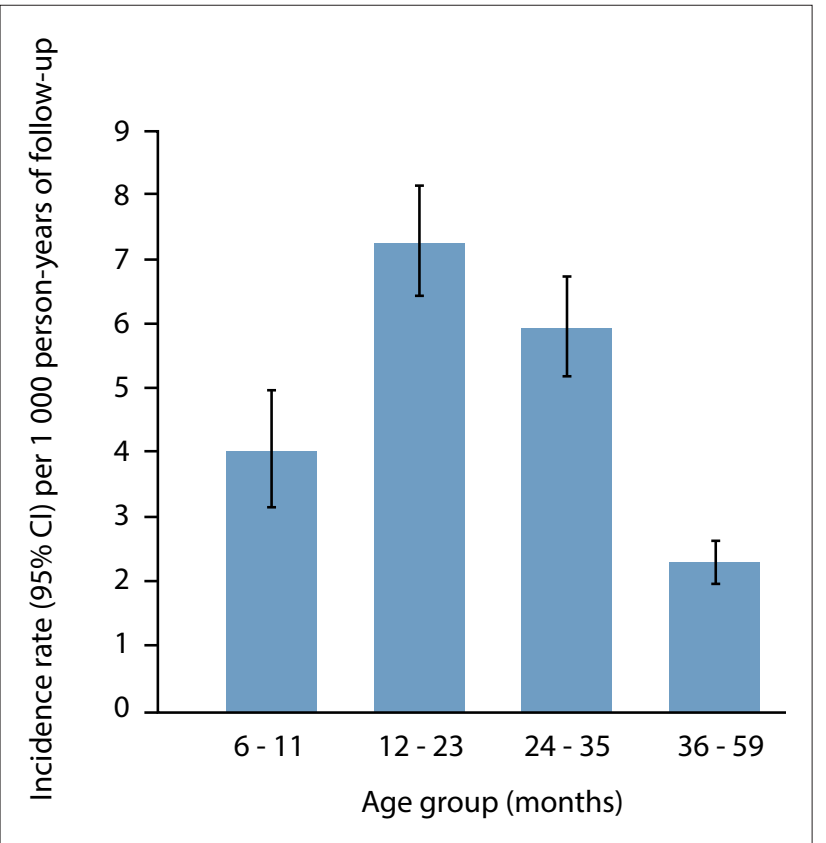

Fig. 2. Incidence rates (95\% CI) per 1000 person-years of follow-up for FS hospitalisations among children 6 - 59 months of age in Soweto, South Africa. The person time contributed by each child was calculated from the age of 6 months and censored on the 5th birthday or death, whichever occurred first. Multiple FSs in one child were counted as separate events if they occurred at least 14 days apart. $(C I=$ confidence interval; $F S=$ febrile seizure. $)$

was the most common virus identified (58.8\%; $n=419 / 713$ ) (Fig. 3B). Children with FS were 2.22-fold (95\% CI 1.56 - 3.16) more likely to be 


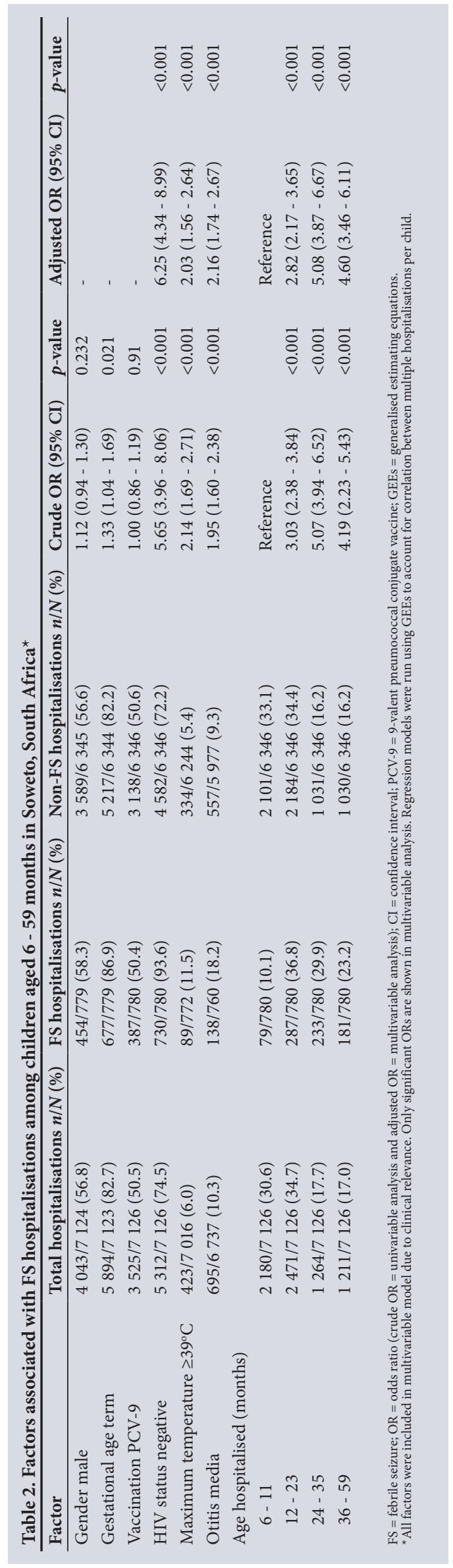

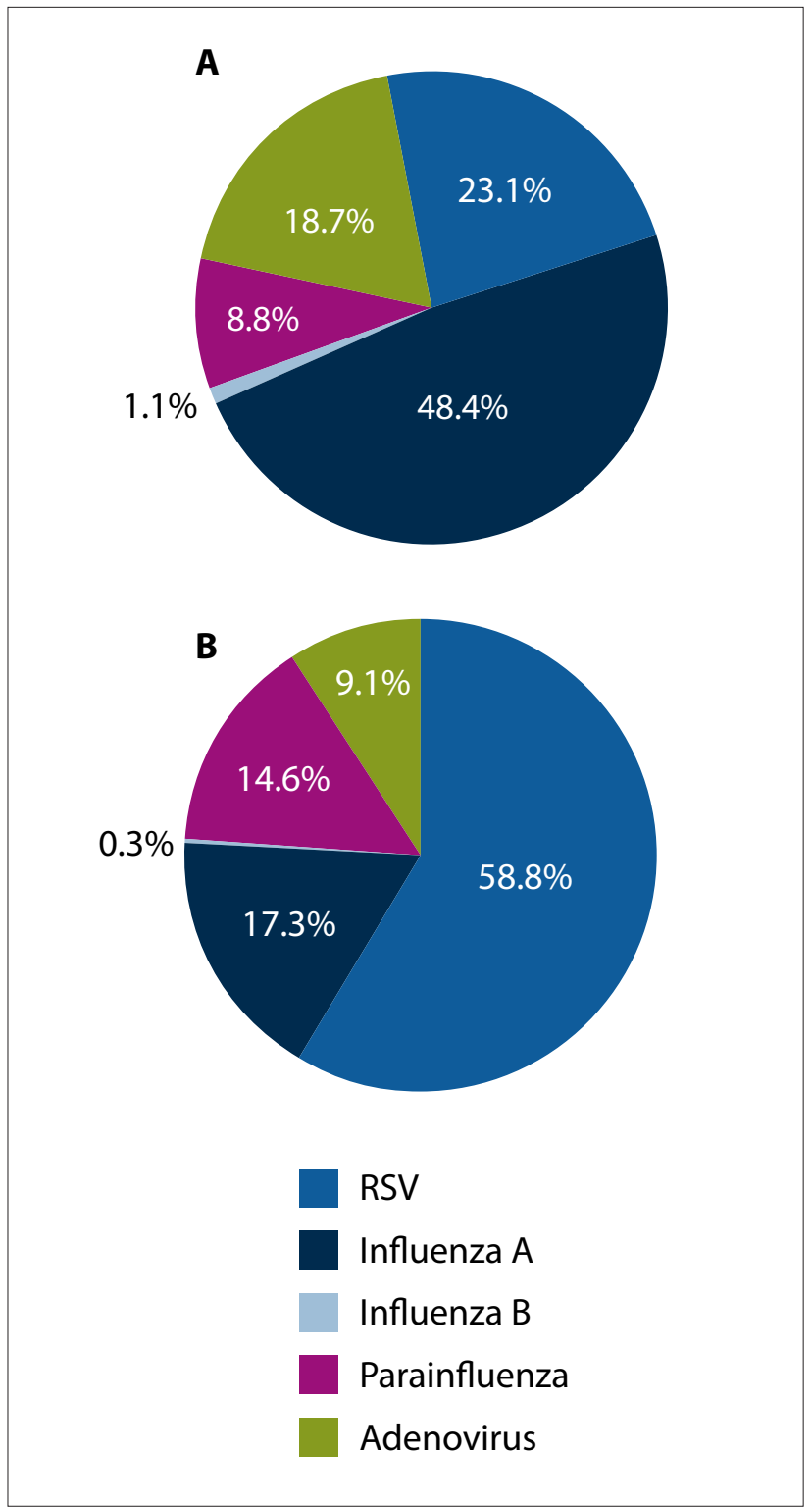

Fig. 3. Proportion of respiratory viruses isolated from FS hospitalisations (A) and non-FS hospitalisations (B) among children 6 - 59 months of age. Proportions are of those with a positive NPA result: 91/804 FS hospitalisations, 713/804 non-FS hospitalisations. (RSV = respiratory syncytial virus; $F S=$ febrile seizure; NPA = nasopharyngeal aspirate.)

infected with influenza A, but $64 \%$ less likely to be infected with RSV (OR 0.36; 95\% CI 0.24 - 0.54) (Table 3) compared with those who had not been hospitalised for FS. The number of FS hospitalisations started increasing in May (mid-autumn), peaked around July (winter) and declined by October (spring) (Fig. 4). A temporal trend was observed for influenza A-associated hospitalisations and FS admission for most years.

\section{Discussion}

FSs were a common cause of paediatric hospitalisation, accounting for almost $11 \%$ of total hospitalisations of children aged $6-59$ months in Soweto. We have shown that respiratory viruses are commonly identified in children hospitalised for FSs in this population, highlighting the role of viral pathogens. Influenza A accounted for the majority of the viruses, which is in agreement with findings from previous studies. ${ }^{[22]}$ Importantly, we showed that influenza A was 
Table 3. Association between respiratory viruses and FS hospitalisations in children 6 - 59 months of age in Soweto, South Africa

\begin{tabular}{|c|c|c|c|c|c|}
\hline Respiratory virus & Total, $n / N(\%)$ & FS, $n / N(\%)$ & Non-FS, $n / N(\%)$ & OR (95\% CI) & $p$-value \\
\hline RSV & $440 / 4309(10.2)$ & $21 / 515(4.1)$ & $419 / 3794(11.0)$ & $0.36(0.24-0.54)$ & $<0.001$ \\
\hline Influenza A & $167 / 4309$ (3.9) & $44 / 515(8.5)$ & $123 / 3794(3.2)$ & $2.22(1.56-3.16)$ & $<0.001$ \\
\hline Influenza B & $3 / 4309(0.07)$ & $1 / 515(0.2)$ & $2 / 3794(0.1)$ & $3.26(0.29-36.09)$ & 0.33 \\
\hline Parainfluenza & $112 / 4309(2.6)$ & $8 / 515(1.6)$ & $104 / 3794(2.7)$ & $0.63(0.33-1.19)$ & 0.15 \\
\hline Adenovirus & $82 / 4309$ (1.9) & $17(3.3)$ & $65 / 3794(1.7)$ & $1.65(0.9-2.79)$ & 0.06 \\
\hline
\end{tabular}

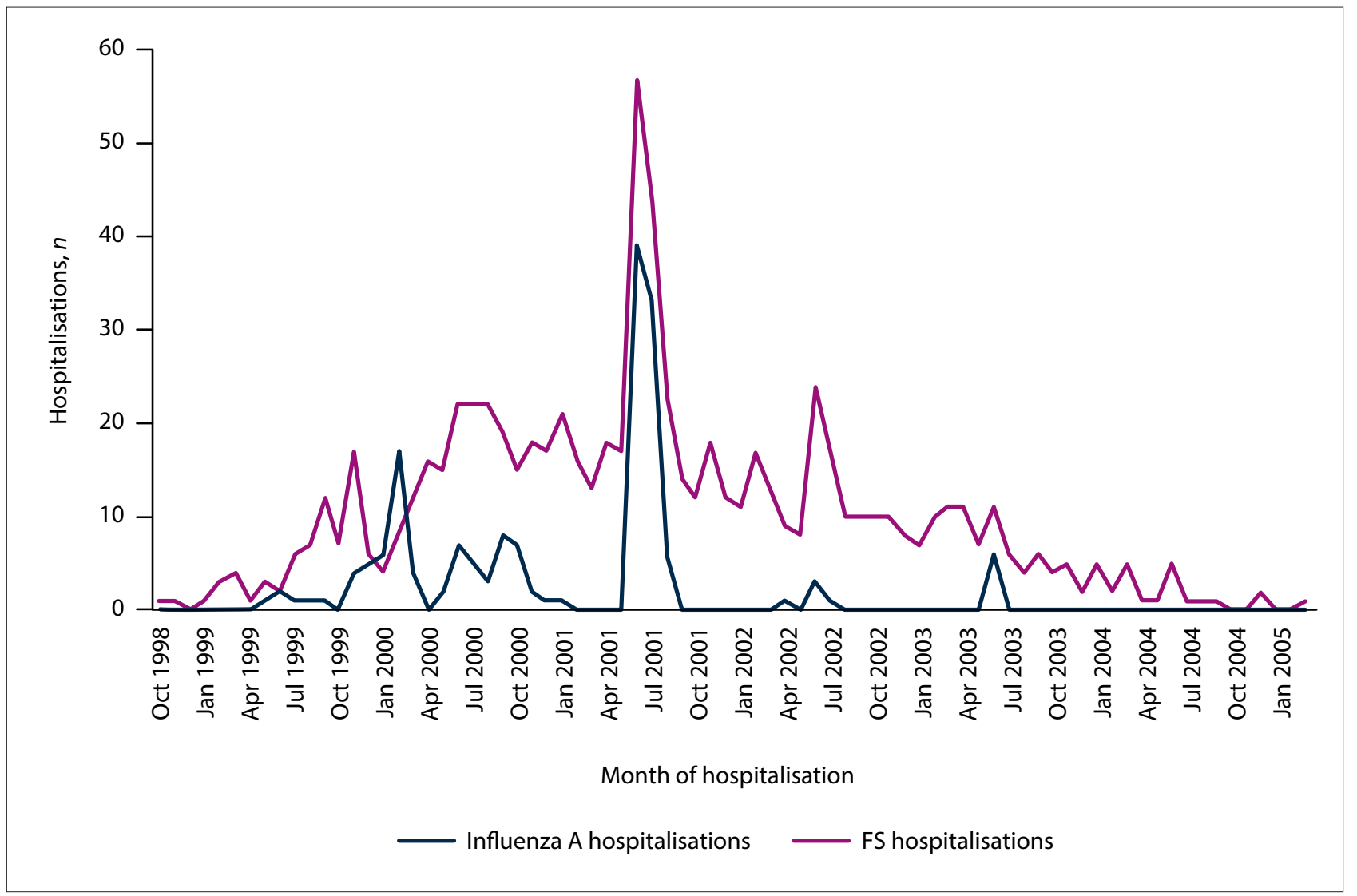

Fig. 4. Distribution of all FS and influenza A hospitalisations over time in children aged $6-59$ months in Soweto, South Africa. (FS = febrile seizure.)

two-fold more likely to be identified in children hospitalised for FSs than in those without FSs. Our results correspond with those of Chiu et al. ${ }^{[10]}$ who analysed medical records of children aged 6 - 60 months hospitalised for respiratory infection in the paediatric department of a university teaching hospital in Hong Kong. Influenza A infection made the greatest contribution to FS hospitalisations and increased the odds of FS hospitalisation by two-fold (OR 1.97). The increased association observed between influenza $\mathrm{A}$ infection and FSs is not well understood. Apart from the fact that influenza infection causes high fever, there have also been reports on the neurotropic properties of influenza A, which may explain the other identified neurological complications associated with influenza A, including acute encephalitis and encephalopathy. ${ }^{[23]}$

The number of FS hospitalisations increased during the winter months in SA, which was temporally associated with the seasonality of influenza A infections. This finding corresponds with Chiu et al. ${ }^{[10]}$ who also described that in contrast to other respiratory viruses, influenza A seasonality was associated with FS hospitalisations. This finding suggests that the seasonal changes in FS hospitalisations may possibly be attributed to influenza A infections, although a more detailed evaluation would be required to assess this with adjustment for confounders.

The overall incidence of FS hospitalisations in Soweto was 4.40 per 1000 person-years. A comparison of this estimate with other studies may be challenging because studies differ according to the methods used for case ascertainment and also by the age definitions. In a Finnish study, ${ }^{[24]}$ the estimated annual incidence of FSs was 14 per 1000 person-years in children followed from birth until the age of 5 years. Cases were identified through diaries completed by parents at home and regular clinic visits, while in our study, cases were identified at the point of hospitalisation and we excluded children aged $<6$ months. In two hospital-based studies conducted in Tanzania and Zambia, the estimated hospital-based incidence of FSs was 37.9 per 1000 and 26.5 per 1000 , respectively. ${ }^{[19,25]}$ The high incidences were in part due to the inclusion of seizures caused by malaria and also including children aged $>5$ years.

The majority of studies showed that FSs occurred more frequently between the ages of 6 and 36 months, with the peak incidence at 18 months. ${ }^{[13,14,26]}$ Similarly, in our study, $60 \%$ of FS hospitalisations occurred between the ages of 12 and 35 months, with the highest 
incidence rates observed at ages $12-23$ months. In our study, $14 \%$ of children who were hospitalised once for FSs had recurrent hospitalisations. This is lower than in previous studies, where estimates of 27 - 35\% have been reported. ${ }^{[26,27]}$ Our study also showed that only children who had their first FS before the age of 36 months experienced more than two FSs before the age of 5 years, and this finding is consistent with previous reports. ${ }^{[13,28]}$

We also found that a concurrent diagnosis of otitis media was associated with two-fold higher odds of FS hospitalisation, and this is comparable to previous literature. In a hospital-based, matched casecontrol study in the USA that investigated factors associated with FSs, children with an underlying illness of acute otitis media were 1.8 times more likely to experience a FS compared with those without. ${ }^{[6]}$

Studies have demonstrated an association between FSs and bacterial infections such as occult bacteraemia, bacterial otitis media and pneumonia. ${ }^{[29,30]}$ Furthermore, viral-bacterial co-infection is common, and PCV-9 has been shown to reduce virus-associated pneumonia caused by influenza A, RSV, parainfluenza and adenovirus. ${ }^{[31]}$ It may therefore be possible for PCV-9 to indirectly reduce the incidence of FS hospitalisations. We did not find evidence to support this hypothesis, as there was no significant difference in the incidence rates of FS hospitalisation between the PCV-9 and placebo groups.

\section{Study limitations}

This analysis has some limitations. Firstly, there may have been some inaccuracies in estimating the total person-years that each child contributed during the follow-up period. Children may have relocated to a different area, died at home or at another health facility, or been lost to follow-up. It is also possible that a proportion of FS cases may have been managed at other private health facilities in the area. This would have underestimated the incidence rate. It is also likely that the investigation and management of FSs would have changed since enrolment of participants in the primary study two decades ago.

Another limitation was that the data collection in the primary study was designed to capture the main study outcome (pneumococcal disease) and not FSs. This limited the selection of variables to be included in our regression model and also affected the interpretation of results. Our regression model suggests that testing HIV-positive was protective against FS hospitalisations; however, the HIV status of children was not measured for the entire cohort in the model, and this therefore may not be a true reflection of the community prevalence of HIV. To our knowledge, there has been no study that has investigated the association between HIV status and FSs, and this warrants further investigation. Similarly, only select respiratory viruses were investigated, and only for children with suspected LRTI. This not only represents a specific subgroup, but it also excluded other viruses previously shown to be strongly associated with FSs, such as rotavirus and HHV-6. Other important variables associated with FSs that were not assessed include a family history of FSs. A history of FSs in a firstdegree family member significantly increases the risk of occurrence of a first FS and also of recurrent FSs. ${ }^{[32]}$ There was also no description of the seizures, i.e. whether they were generalised or focal or whether they occurred multiple times within a period of 24 hours. This information would have allowed for the distinction between simple and complex FSs.

\section{Conclusions}

FSs were a common cause of paediatric hospitalisation at a tertiary hospital in Soweto, SA. The highest incidence was observed in children 12 - 35 months of age. Detection of influenza A was significantly associated with FSs among hospitalised children, doubling the odds of hospitalisation for FSs. In particular, the role of respiratory viruses in the pathogenesis of FSs warrants further investigation. There are licensed influenza vaccines available that may potentially have an impact on the incidence of FSs, providing additional benefits to their use.

Declaration. The research for this study was done in partial fulfilment of the requirements for NDT's MSc (Epidemiology) degree at the University of the Witwatersrand.

Acknowledgements. None.

Author contributions. NDT (corresponding author) was involved in the design of the research, formal analysis and interpretation of data as well as drafting the manuscript. MJG and ZD were involved in the conceptualisation and design of the research as well as supervising the methodology process, editing and approving the version to be submitted. SAM and CC played a role in approving the design as well as editing and approving the version to be submitted.

Funding. None.

Conflicts of interest. None.

1. Krumholz A, Grufferman S, Orr ST, Stern BJ. Seizures and seizure care in an emergency department. Epilepsia 1989;30(2):175-181. https://doi.org/10.1111/j.1528-1157.1989.tb05451.x

2. Chen CY, Chang YJ, Wu HP. New-onset seizures in pediatric emergency. Pediatr Neonatol 2010;51(2):103-111. https://doi.org/10.1016/s1875-9572(10)60019-8

3. Smith RA, Martland T, Lowry MF. Children with seizures presenting to accident and emergency. 3. Smith RA, Martland T, Lowry MF. Children with seizures presenting to
J Accid Emerg Med 1996;13(1):54-58. https://doi.org/10.1136/emi.13.1.54

J Accid Emerg Med 1996;13(1):54-58. https://doi.org/10.1136/emj.13.1.54
4. Friedman MI, Sharieff GQ. Seizures in children. Pediatr Clin North Am 2006;53(2):257-277. https:// 4. Friedman MJ, Sharieff GQ. Seizure
doi.org/10.1016/j.pcl.2005.09.010

5. American Academy of Pediatrics. Febrile seizures: Clinical practice guideline for the long-term management of the child with simple febrile seizures. Pediatrics 2008;121(6):1281-1286. https://doi. org/10.1542/peds.2008-0939

6. Berg AT, Shinnar S, Shapiro ED, Salomon ME, Crain EF, Hauser WA. Risk factors for a first febrile seizure: A matched case-control study. Epilepsia 1995;36(4):334-341. https://doi. org/10.1111/j.1528-1157.1995.tb01006.x

7. Vestergaard M, Basso O, Henriksen TB, Ostergaard JR, Olsen J. Risk factors for febrile convulsions. Epidemiology 2002;13(3):282-287. https://doi.org/10.1097/00001648-200205000-00008

8. Laina I, Syriopoulou VP, Daikos GL, et al. Febrile seizures and primary human herpesvirus 6 infection. Pediatr Neurol 2010;42(1):28-31. https://doi.org/10.1016/j.pediatrneurol.2009.07.016

9. Lloyd MB, Lloyd JC, Gesteland PH, Bale JF jr. Rotavirus gastroenteritis and seizures in young children. 9. Lloyd MB, Lloyd JC, Gesteland PH, Bale JF jr. Rotavirus gastroenteritis and seizures in yo
Pediatr Neurol 2010;42(6):404-408. https://doi.org/10.1016/j.pediatrneurol.2010.03.002

10. Chiu SS, Tse CY, Lau YL, Peiris M. Influenza A infection is an important cause of febrile seizures. Pediatrics 2001;108(4):1004-1005. https://doi.org/10.1542/peds.108.4.e63

11. Kolahi AA, Tahmooreszadeh S. First febrile convulsions: Inquiry about the knowledge, attitudes and concerns of the patients' mothers. Eur J Pediatr 2009;168(2):167-171. https://doi.org/10.1007/s00431008-0724-z

12. Ling SG. Parental response and understanding towards febrile convulsion. Med J Malaysia 2000;55(4):419-423.

13. Verity CM, Butler NR, Golding J. Febrile convulsions in a national cohort followed up from birth. IPrevalence and recurrence in the first five years of life. BMJ (Clin Res Ed) 1985;290(6478):1307-1310. https://doi.org/10.1136/bmj.290.6478.1307

14. Vestergaard M, Christensen J. Register-based studies on febrile seizures in Denmark. Brain Dev 2009;31(5):372-377. https://doi.org/10.1016/j.braindev.2008.11.012

15. Hackett R, Hackett L, Bhakta P. Febrile seizures in a south Indian district: Incidence and associations. Dev Med Child Neurol 1997;39(6):380-384. https://doi.org/10.1111/j.1469-8749.1997.tb07450.x

16. Storz C, Meindl M, Matuja W, Schmutzhard E, Winkler AS. Community-based prevalence and clinical characteristics of febrile seizures in Tanzania. Pediatr Res 2015;77(4):591-596. https://doi.org/10.1038/ characteris
pr.2015.3

pr.2015.3
17. Idro R, Gwer S, Kahindi M, et al. The incidence, aetiology and outcome of acute seizures in children . Idro R, Gwer S, Kahindi M, et al. The incidence, aetiology and outcome of acute seizures in children
admitted to a rural Kenyan district hospital. BMC Pediatr 2008;8:5. https://doi.org/10.1186/14712431-8-5

18. Kariuki SM, Ikumi M, Ojal J, et al. Acute seizures attributable to falciparum malaria in an endemic area on the Kenyan coast. Brain 2011;134(5):1519-1528. https://doi.org/10.1093/brain/awr051

9. Winkler AS, Tluway A, Schmutzhard E. Febrile seizures in rural Tanzania: Hospital-based incidence and clinical characteristics. J Trop Pediatr 2013;59(4):298-304. https://doi.org/10.1093/tropej/fmt022

20. Klugman KP, Madhi SA, Huebner RE, Kohberger R, Mbelle N, Pierce N. A trial of a 9-valent pneumococcal conjugate vaccine in children with and those without HIV infection. N Engl J Med 2003;349(14):1341-1348. https://doi.org/10.1056/nejmoa035060

21. Zeger SL, Liang KY, Albert PS. Models for longitudinal data: A generalised estimating equation approach. Biometrics 1988;44(4):1049-1060. https://doi.org/10.2307/2531734

22. Chung B, Wong V. Relationship between five common viruses and febrile seizure in children. Arch Dis Child 2007;92(7):589-593. https://doi.org/10.1136/adc.2006.110221
.

23. Newland JG, Laurich VM, Rosenquist AW, et al. Neurologic complications in children hospitalised with influenza: Characteristics, incidence, and risk factors. J Pediatr 2007;150(3):306-310. https://doi.
witise with influenza: Characteristics, in
org/10.1016/.jpeds.2006.11.054

24. Sillanpaa M, Camfield P, Camfield C, et al. Incidence of febrile seizures in Finland: Prospective population-based study. Pediatr Neurol 2008;38(6):391-394. https://doi.org/10.1016/j.
pediatrneurol.2008.02.006 
25. Birbeck GL. Seizures in ruralZambia. Epilepsia 2000;41(3):277-281.https://doi.org/10.1111/j.1528-1157.2000. tb00156.x

26. Berg AT, Shinnar S, Hauser WA, et al. A prospective study of recurrent febrile seizures. N Engl J Med 1992;327(16):1122-1127. https://doi.org/10.1056/nejm199210153271603

27. Van Esch A, Steyerberg EW, Berger MY, Offringa M, Derksen-Lubsen G, Habbema JD. Family history and recurrence of febrile seizures. Arch Dis Child 1994;70(5):395-399. https://doi.org/10.1136/adc.70.5.395

28. Capovilla G, Mastrangelo M, Romeo A, Vigevano F. Recommendations for the management of febrile seizures': Ad Hoc Task Force of LICE Guidelines Commission. Epilepsia 2009;50(Suppl 1):2-6. https:// doi.org/10.1111/j.1528-1167.2008.01963.x

29. McIntyre P, Harris F. Occult pneumococcal bacteraemia and febrile convulsions. BMJ (Clin Res Ed) 1983;286(6370):1055-1056. https://doi.org/10.1136/bmj.286.6360.203
30. Shah SS, Alpern ER, Zwerling L, Reid JR, McGowan KL, Bell LM. Low risk of bacteremia in children with febrile seizures. Arch Pediatr Adolesc Med 2002;156(5):469-472. https://doi.org/10.1001/ archpedi.156.5.469

31. Madhi SA, Klugman KP. A role for Streptococcus pneumoniae in virus-associated pneumonia. Nat Med 2004;10(8):811-813. https://doi.org/10.1038/nm1077

32. Sharawat IK, Singh J, Dawman L, Singh A. Evaluation of risk factors associated with first episode febrile . Sharawat IK, Singh J, Dawman L, Singh A. Evaluation of risk factors associated with first episode febr
seizure. J Clin Diagn Res 2016;10(5):SC10-SC13. https://doi.org/10.7860/JCDR/2016/18635.7853

Accepted 26 February 2021 